\section{anthropology} \& materialism

\section{Anthropology \& Materialism}

A Journal of Social Research

1 | 2013

Across the Fields

\title{
Walter Benjamin et le surréalisme
}

Les noces chimiques de deux matérialismes

Walter Benjamin and Surrealism: the Chimical Nuptials of two Materialisms

Walter Benjamin y el surrealismo. Las nupcias químicas de dos materialismos

\section{Michael Löwy}

\section{OpenEdition \\ Journals}

\section{Édition électronique}

URL : http://journals.openedition.org/am/123

DOI : 10.4000/am.123

ISSN : 2364-0480

\section{Éditeur :}

CETCOPRA, CRASSH - Center for Research in the Arts Social Sciences and Humanities, Fakultät Gestaltung - Universität der Künste Berlin

Référence électronique

Michael Löwy, «Walter Benjamin et le surréalisme », Anthropology \& Materialism [En ligne], 1 | 2013, mis en ligne le 30 octobre 2014, consulté le 04 mai 2019. URL : http://journals.openedition.org/am/123 ;

DOI : 10.4000/am. 123

Ce document a été généré automatiquement le 4 mai 2019.

Tous droits réservés 


\title{
Walter Benjamin et le surréalisme
}

\author{
Les noces chimiques de deux matérialismes \\ Walter Benjamin and Surrealism: the Chimical Nuptials of two Materialisms \\ Walter Benjamin y el surrealismo. Las nupcias químicas de dos materialismos
}

\section{Michael Löwy}

\section{Introduction}

1 Qu'ont en commun Georg Büchner, Ludwig Feuerbach, Jean Paul, Karl Gutzkow, Gottfried Keller, Prosper Enfantin, Claire Démar, Charles Fourier et les surréalistes ? Ce sont, parmi d'autres, des exemples de matérialisme anthropologique selon Walter Benjamin. Ce n'est pas dans les habitudes de ce penseur subtil et elliptique de donner des définitions cartésiennes de ses concepts : leur richesse polysémique est d'ailleurs une des raisons du charme qu'exercent ses écrits... L'expression qu'il utilise au sujet de Gottfried Keller, " athéisme hédoniste », est une piste intéressante, mais bien insuffisante ${ }^{1}$. La même chose vaut pour l'émancipation de la chair d'Enfantin, le féminisme radical de Claire Démar, ou l'harmonie des passions chez Fourier.

2 Je me garderai bien de proposer une définition, mais je risquerai l'hypothèse suivante : le matérialisme anthropologique pourrait être considéré comme une des manifestations d'un imaginaire romantique critique et/ou utopique, en rupture avec la religion, et/ou avec l'idéalisme allemand et/ou avec le matérialisme vulgaire. Par « romantisme», je n'entends pas ici seulement une école littéraire du XIX siècle, mais une vision du monde, une protestation culturelle contre le désenchantement capitaliste du monde, contre la civilisation bourgeoise moderne, au nom de valeurs pré-capitalistes. D'une certaine façon, le romantisme peut être considéré comme une tentative, animée par l'énergie du désespoir et éclairée par le soleil noir de la mélancolie (Gérard de Nerval), de réenchantement du monde, sous des formes religieuses (chez les romantiques traditionalistes) ou profanes (chez les «matérialistes anthropologiques $»^{2}$ ). Une révolte « moderne / antimoderne » qui peut prendre des formes régressives - le rêve d'un impossible retour au passé - ou des formes critiques/utopiques, aspirant à un détour par le passé, en direction 
d'un avenir nouveau, libre et fraternel. Bien entendu, c'est à cette deuxième sensibilité qu'appartiennent aussi bien Walter Benjamin que les surréalistes.

\section{Non-conformisme moral et révolution prolétarienne}

Dans une note du Livre des Passages Benjamin cite un texte d'Emmanuel Berl - un rationaliste borné, hostile aux surréalistes - qui dénonce dans leurs écrits une confusion entre «le non-conformisme moral et la révolution prolétarienne », typique de la période antérieure à Marx, celle du socialisme utopique des années 1820-1840. Benjamin se dissocie de cette position, et observe, chez les surréalistes, des éléments qui semblent "réfractaires au marxisme »: le matérialisme anthropologique et l'hostilité au progrès. Or, nous savons que le marxisme que Benjamin se propose de reconstruire est précisément fondé sur l'inclusion de ces deux éléments. Ainsi, dans une autre note du même livre, où il présente Enfantin, Büchner et Feuerbach comme des représentants du matérialisme anthropologique, il esquisse la conclusion suivante: «Le matérialisme dialectique inclut le matérialisme anthropologique $»^{3}$.

4 Cette "inclusion ", ou articulation, ou fusion entre les deux matérialismes est facilitée par les affinités électives qu'ils entretiennent : non seulement le matérialisme et l'athéisme, mais aussi la révolte contre l'ordre bourgeois - famille, État, propriété privée - et l'utopie d'une nouvelle société. Cette convergence est un des thèmes centraux de l'essai de 1929, "Le surréalisme, dernier instantané de l'intelligentsia européenne », un des écrits les plus « illuminés » (profanes) que Benjamin ait jamais rédigé. Je reprends ici à mon compte ce qu'a avancé Marc Berdet dans sa thèse : l'objectif de Benjamin est de corriger le matérialisme dialectique par le matérialisme anthropologique, et inversement, en vue de « redonner une épaisseur sensible à la révolution sans lui ôter sa vertu émancipatrice » ${ }^{4}$.

\section{Rimbaud avec Marx}

Dans un passage révélateur de l'article sur le surréalisme, Benjamin oppose le matérialisme anthropologique de Hebel, Büchner, Nietzsche, Rimbaud et des surréalistes - encore une configuration quelque peu différente de celle du Livre des Passages - au matérialisme métaphysique de Vogt et de Boukharine ${ }^{5}$. Si Karl Vogt et Jacobus Moleschott incarnent depuis toujours, aux yeux des marxistes, le matérialisme mécaniciste, évolutionniste, métaphysique et anti-dialectique du XIX ${ }^{\mathrm{e}}$ siècle, la référence à Nikolai Ivanovitch Boukharine, un des principaux penseurs du marxisme soviétique des années 1920, est bien plus irrévérente. Elle témoigne de l'indépendance d'esprit de Benjamin, malgré sa sympathie pour l'expérience soviétique et le mouvement communiste, clairement affichée à partir de 1924. On peut se demander si Benjamin n'a pas lu la brillante critique du matérialisme positiviste de Boukharine publiée en 1925 par Georg Lukacs. Cette prise de distance assez nette envers le marxisme soviétique explique peutêtre l'étonnante conclusion politique de l'article : «les surréalistes sont les seuls à avoir compris l'ordre qu'il [NB: le Manifeste Communiste] nous donne aujourd'hui $»^{6}$. Est-ce grâce à l'apport du matérialisme anthropologique? En tout cas, les surréalistes apparaissent, aux yeux de Benjamin, comme les véritables héritiers de Marx et du programme communiste. 
6 La référence à Rimbaud - un des grands précurseurs de leur mouvement, aux yeux des surréalistes eux-mêmes - est très significative. Elle peut surprendre : Marx et Rimbaud, le sobre critique de l'économie politique, et le poète magique du Bateau ivre sont-ils compatibles? En les associant, dans un même paragraphe, Benjamin devance ici André Breton, qui proposera - quelques années plus tard - dans son discours au Congrès des Ecrivains contre le fascisme (juin 1935) la formule suivante :

«'Transformer le monde' a dit Marx ; 'changer la vie' a dit Rimbaud : ces deux mots

d'ordre pour nous n'en font qu'un. $»^{7}$

7 Dans ce brillant raccourci du programme surréaliste, peut-être sa définition la plus réussie, il n'est plus question de convergence ou d'analogie, mais tout simplement d'une fusion, d'une "noce chimique» - expression alchimique qu'affectionnaient les surréalistes - entre une des principales figures de ce que Benjamin appelle le matérialisme anthropologique, et la pensée marxiste. Benjamin se réjouit de la politisation du surréalisme, de son adhésion au programme marxiste, mais cela ne signifie pas, à ses yeux, qu'il doive renoncer à sa charge poétique et rebelle, bien au contraire :

«Gagner à la révolution les forces de l'ivresse, c'est à quoi tend le surréalisme dans tous ses livres et dans toutes ses entreprises? C'est ce qu'il est en droit d'appeler sa tâche la plus spécifique. $»^{8}$

8 En quoi consiste donc cette ivresse, ce Rausch dont les surréalistes seraient les porteurs par excellence? Bien entendu, il ne s'agit pas de celle qui résulte de l'excès de boissons alcoolisées... Dans Sens Unique (1928), Benjamin se réfère à l'ivresse comme à l'expression $\mathrm{du}$ rapport magique de l'homme ancien au cosmos, mais il laisse entendre que l'expérience (Erfahrung) du Rausch qui caractérisait cette relation rituelle avec le monde a disparu de la société moderne. Or, dans l'essai de 1929, il semble l'avoir retrouvée, sous une forme nouvelle, dans le surréalisme.

9 Benjamin distingue, dans cet écrit, les basses manifestations de l'ivresse - « les extases de la religion ou de la drogue ", qui sont d'ailleurs très proches, puisque « la religion est l'opium du peuple» - et ses formes supérieures, qui relèvent du matérialisme anthropologique :

«Le dépassement créateur de l'illumination religieuse ne se trouve pas dans les stupéfiants. Il se trouve dans une illumination profane, dans une inspiration matérialiste, anthropologique [...]. ${ }^{9}$

10 Le concept d' « illumination profane » n'est pas facile à cerner, mais il renvoie sans doute à l'éclat, au scintillement, à l'incandescence de la tentative surréaliste - typiquement romantique - de ré-enchantement du monde. Une tentative qui est radicalement profane parce que rien n'est aussi abominable, aux yeux des surréalistes, que la religion en général et la religion catholique apostolique romaine en particulier; voici pourquoi Benjamin insiste sur « la révolte amère et passionnée contre le catholicisme dans laquelle Rimbaud, Lautréamont, Apollinaire ont donné naissance au surréalisme $»^{10}$.

11 Curieusement, Benjamin reproche aux surréalistes « une conception trop courte, non dialectique, de la nature de l'ivresse ». Ils ne se rendent pas compte que la lecture et la pensée sont elles aussi de l'illumination profane; par exemple, "l'étude la plus passionnée de l'ivresse du hachisch ne nous apprendra pas sur la pensée (qui est un éminent narcotique) la moitié de ce que cette illumination profane qu'est la pensée nous apprend sur l'ivresse du hachisch $»^{11}$. Cette critique est d'autant plus étrange que les surréalistes - contrairement à Benjamin (voir son texte « Hachisch à Marseille ») ! - n'ont jamais été très portés sur les expériences avec les drogues, et ont toujours manifesté plus 
d'intérêt pour les Mémoires d'un mangeur d'opium de Thomas de Quincey que pour la consommation de ce doux narcotique.

Parmi les illuminations profanes d'inspiration matérialiste anthropologique admirées par Breton et par Benjamin, il y en a une qui est tout à fait dans l'esprit de ce romantisme utopique - ou de ce "marxisme gothique ", pour employer un concept proposée par une historienne du surréalisme, Margaret Cohen ${ }^{12}$ - dont nous avons parlé plus haut : l'amour courtois du Moyen-Age. Cet amour, auquel Breton rend hommage dans Nadja, ressemble, selon l'historien de la culture Erich Auerbach cité par Benjamin, « plus à une illumination qu'à une jouissance sensible ", c'est-à-dire, selon Benjamin, à une forme d'ivresse ou de « ravissement ${ }^{13}$.

\section{Vers un communisme libertaire}

La convergence entre les deux matérialismes - le dialectique et l'anthropologique - dans le surréalisme, trouve dans l'essai de 1929 une expression directement politique: la convergence - et la correction mutuelle - du communisme et de l'anarchisme. Le rapport des surréalistes au deuxième est présenté par Benjamin dans les termes suivants: «Depuis Bakounine, l'Europe ne disposait plus d'une idée radicale de la liberté. Les surréalistes ont cette idée $»^{14}$. Dans la vaste littérature sur le surréalisme des soixante-dix dernières années, il est rare de trouver une formule aussi évocatrice, aussi capable d'exprimer, par la grâce de quelques mots simples et tranchants, le « noyau infracassable de nuit » (Breton) de ce mouvement.

Benjamin considère donc que " gagner à la révolution les forces de l'ivresse » - dont nous avons vu les liens intimes avec le matérialisme anthropologique - est la grande tâche politique du surréalisme. Mais il ajoute immédiatement cette réserve :

« Pour y arriver, il ne suffit pas que tout acte révolutionnaire comporte, comme nous le savons, une part d'ivresse. Mais y insister de façon exclusive serait négliger entièrement la préparation méthodique et disciplinée de la révolution au profit d'une pratique qui oscille entre l'exercice et la célébration anticipée. $»^{15}$

En d'autres termes : il faut pouvoir combiner l'ivresse et la discipline, l'anarchisme et le communisme, de façon à permettre une correction réciproque entre les deux. Cette orientation est d'ailleurs celle de Benjamin lui-même, qui se présente, dans les premiers paragraphes de l'essai comme un « observateur allemand » qui a «éprouvé dans sa chair l'extrême vulnérabilité de cette position entre fronde anarchiste et discipline révolutionnaire $»^{16}$. Comme l'on sait, les surréalistes, et André Breton en particulier, se situeront eux-mêmes dans cette position vulnérable, d'abord dans les marges du Parti Communiste, puis, après 1935, en coopérant avec Trotsky et l'opposition de gauche, avant de tenter, dans les années 1951-53, une collaboration avec les communistes libertaires (anarchistes) ${ }^{17}$.

\section{Au bord du mythe}

Reste à savoir si le matérialisme anthropologique et les « forces de l'ivresse » ne risquent pas de sombrer dans le mythe. Voici comment Marc Berdet pose la question dans sa thèse: "La frontière est ténue entre l'utopie et le mythe, entre le matérialisme anthropologique et son simulacre (...). Comment tracer la frontière sans sacrifier l'utopie 
au mythe $? »^{18}$. En effet, le risque existe, et on peut l'illustrer par un cercle de brillants intellectuels français dont Benjamin était proche, tout en gardant avec eux une distance critique: le Collège de Sociologie, animé par Georges Bataille, Roger Caillois, Pierre Klossowski et Michel Leiris, dont l'attirance pour le mythe est précisément l'une des caractéristiques centrales. Le cas de Georges Bataille, et de ses rapports avec les surréalistes, est particulièrement révélateur. L'ivresse prend chez lui la forme de l'exaltation de la dépense, de l'excès, de la fête, du sacrifice, et de la mort. Proche des surréalistes, il rompra avec Breton en 1930. Une éphémère réconciliation aura lieu en 1935, autour du Manifeste Contre-Attaque, signé par Bataille et Klossowski, ainsi que par Breton, Éluard et les autres surréalistes.

L'objectif de ce document était de rassembler les intellectuels révolutionnaires, les partisans de la lutte de classes contre le fascisme, mais il inclut quelques formules dangereuses, qui suggèrent une sorte d'étrange fascination pour l'ennemi: «nous entendons (...) nous servir des armes crées par le fascisme, qui a su utiliser l'aspiration fondamentale des hommes à l'exaltation affective et au fanatisme ${ }^{19}$. Quelques mois plus tard, peu à l'aise avec cet argumentaire inspiré par Bataille, les surréalistes quitteront «Contre Attaque » pour se rapprocher, progressivement, des thèses anti-fascistes plus « classiques » (en termes marxistes) de l'opposition de gauche (trotskyste). Il faut ajouter cependant que non seulement chez les surréalistes mais aussi chez Bataille et ses amis, il n'y aura pas de passage dans le camp du fascisme, que ce soit avant ou pendant la guerre ${ }^{20}$

\section{Conclusion}

La tentative - inachevée - parfaitement hétérodoxe du point de vue des tendances dominantes du marxisme de son époque, de susciter, en s'appuyant sur le surréalisme, une convergence, alliance, alliage, fusion ou inclusion, entre le matérialisme dialectique et le matérialisme anthropologique, entre Marx et Rimbaud - plus tard, dans le Livre des Passages, entre Marx et Fourier ${ }^{21}$ - dans une perspective de complémentarité et de correction réciproque, est une des aventures intellectuelles les plus insolites et les plus fascinantes de l'œuvre de Walter Benjamin.

\section{BIBLIOGRAPHIE}

Benjamin Walter, Euvres II, Paris, Gallimard, 2000

Benjamin Walter, Paris, capitale du XIX ${ }^{\mathrm{e}}$ siècle. Le livre des passages, Paris, Cerf, 1989

Berdet Marc, Mouvement social et fantasmagories dans Paris, capitale du XIXe siècle, Thèse de doctorat à l’Université de Paris 7, juin 2009. Résumé en ligne ici : http://trajectoires.revues.org/ index $280 . h \mathrm{html}$

Breton André, Position politique du surréalisme, Paris, Denoël/Gonthier, 1972 
Cohen Margaret, Profane Illumination. Walter Benjamin and the Paris of Surrealist Revolution, Berkeley, University of California Press, 2003

Löwy Michael, L'étoile du matin. Surréalisme et marxisme, Paris, Syllepse, 2000

Löwy Michael et Sayre Robert, Esprits de feu. Figures de l'anti-capitalisme romantique, Paris, Éditions du Sandre, 2010

Nadeau Maurice, Documents surréalistes, Paris, Seuil, 1948

Thomas François, Rimbaud et Marx : une rencontre surréaliste, Paris, L'Harmattan, 2007

\section{NOTES}

1. Walter Benjamin, « Gottfried Keller » (1927), in CEuvres, II, Paris, Folio Gallimard, 2000, p. 19.

2. Pour une discussion plus détaillée de ce concept, y compris par rapport à Walter Benjamin, je renvoie à mon récent ouvrage, avec Robert Sayre, Esprits de feu. Figures de l'anti-capitalisme romantique, Paris, Editions du Sandre, 2010.

3. Walter Benjamin, Paris, capitale du XIX ${ }^{e}$ siècle. Le livre des Passages, Paris, Cerf, 1989, p. 607, 709. En matière de confusion, Emmanuel Berl est un grand expert : après avoir collaboré avec le fasciste français Georges Vallois, il passe à gauche et soutient le Front Populaire, pour ensuite se rallier au Maréchal Pétain, dont il écrira quelques discours en $1941 . .$.

4. Marc Berdet, Mouvement social et fantasmagories dans Paris, capitale du XIXeme siècle, Thèse de doctorat à l'Université de Paris 7, juin 2009, p. 489.

5. Walter Benjamin, «Le surréalisme, le dernier instantané de l'intelligentsia européenne » (1929), Euvres II, op. cit., p. 134.

6. Ibid.

7. André Breton, «Discours au Congrès des Ecrivains » (1935), Position politique du surréalisme, Paris, Denoël//Gonthier, 1972, p. 95. Voir à ce propos l'intéressant ouvrage de Frédéric Thomas, Rimbaud et Marx : une rencontre surréaliste, Paris, L'Harmattan, 2007.

8. Walter Benjamin, « Le surréalisme », art. cit., p. 130.

9. Ibid., p. 116-117.

10. Ibid., p 116.

11. Ibid., p.131.

12. Margaret Cohen, Profane Illumination. Walter Benjamin and the Paris of Surrealist Revolution, Berkeley, University of California Press, 2003.

13. Walter Benjamin, « Le surréalisme », art. cit., p. 119.

14. Ibid., p 129. Suit une citation de Nadja où André Breton proclame que la liberté « sous sa forme révolutionnaire la plus simple, qui n'est pas moins l'émancipation humaine à tous égards (...) demeure la seule cause qu'il soit digne de servir » (ibid. p. 130).

15. Ibid., p. 130.

16. Ibid., p. 113-114.

17. Voir à ce sujet mon essai «Le marxisme libertaire d'André Breton», in L'étoile du matin. Surréalisme et marxisme, Paris, Syllepse, 2000.

18. Marc Berdet, op. cit., p. 17.

19. «Contre Attaque. Union de lutte des intellectuels révolutionnaires », in Maurice Nadeau, Documents Surréalistes, Paris, Seuil, 1948, p. 320.

20. Salvador Dali pourrait être une exception, mais son ralliement au «monarchisme » et au catholicisme en Espagne franquiste relève plutôt de l'opportunisme que d'un vrai choix politique. 21. Sur ce sujet aussi Benjamin était en avance sur les surréalistes : André Breton ne publiera son Ode à Charles Fourier qu'en 1947... 


\section{RÉSUMÉS}

Cet article interprète le matérialisme anthropologique comme l'expression d'un imaginaire contestataire propre au romantisme anticapitaliste. Chez Walter Benjamin, il s'articule avec le matérialisme dialectique, y introduisant la critique du désenchantement du monde et la revalorisation de l'utopie. Parsemé d'«illuminations profanes», le programme politique du surréalisme est paradigmatique d'une telle alliance.

This article interprets anthropological materialism as the expression of a contestant imaginary peculiar to anticapitalist romanticism. Following Walter Benjamin this is linked to dialectical materialism, introducing within it the critique of the disenchantment of the world and the revaluation of utopia. Scattered with "profane illuminations", the political program of surrealism is paradigmatic of such an alliance.

En este artículo se interpreta el materialismo antropológico como la expresión de un imaginario contestatario propio al romanticismo anticapitalista. En Walter Benjamin, esto se articula con el materialismo dialéctico, al ser introducida la crítica del desencantamiento del mundo y la revalorización de la utopía. Sembrado de «iluminaciones profanas », el programa político del surrealismo aparecerá como el paradigma de dicha alianza.

INDEX

Keywords : materialism, romanticism, utopia, surrealism, Benjamin Walter

Mots-clés : matérialisme, romantisme, utopie, surréalisme, Benjamin (Walter)

Palabras claves : materialismo, romanticismo, utopía, surrealismo, Benjamin Walter

\section{AUTEUR}

MICHAEL LÖWY

Sociologue et philosophe, CNRS 